\title{
Brain-derived neurotrophic factor (BDNF) in stress and affective disorders
}

\author{
Molecular Psychiatry (2002) 7, 519. doi:10.1038/ \\ sj.mp.4001211
}

The role of brain-derived neurotrophic factor (BDNF) in stress and affective disorders has been studied for 10 years. Spina et al. showed in 1992 that BDNF was protective to neurons in conditions of chemical stress. ${ }^{1}$ In 1995 Mark Smith and colleagues showed that the expression of BDNF in the brain was regulated by stress and suggested a role for BDNF in recurrent depression, aging, and posttraumatic stress disorder. ${ }^{2}$ ${ }^{3}$ Later that year, Ron Duman's group showed that BDNF was downregulated by antidepressant treatments that included various classes of antidepressant drugs and chronic electroconvulsive seizures. In 1997 Ron Lindsay's group showed that BDNF treatment reversed two animal paradigms that have been used as models of depression, namely learned helplessness following exposure to inescapable shock and the forced swim test. Those findings were replicated by Ron Duman's group in $2002 .^{4}$

The mechanisms for the effects of BDNF have also been studied: Frerking et al have shown that BDNF causes a significant decrease in stimulus-evoked inhibitory postsynaptic currents (IPSCs) on hippocampal CA1 pyramidal cells. ${ }^{5}$ Additionally, Sherwood and Lo have shown that chronic BDNF treatment induced long-term (days to weeks) enhancement of the strength of non-NMDA receptor-mediated glutamatergic transmission. In that study, chronic BDNF treatment also decreased synaptic depression.

Human studies have provided preliminary data that support a role for BDNF in affective disorders: Chen et al have documented increased BDNF immunoreactivity in postmortem hippocampal tissue obtained from subjects treated with antidepressant medications at the time of death, compared with antidepressant-untreated subjects. ${ }^{6}$ Karege et al have shown decreased serum BDNF levels in 30 depressed subjects diagnosed with major depression. ${ }^{7}$

The role of BDNF in mood disorders is further strengthened by two papers published in this issue of Molecular Psychiatry. Maternal deprivation is a paradigm that is thought to be relevant to depression. In 1999 we published an article by Bernard Lerer's group in which it was shown that in humans, early parental loss (loss of a parent before age 9) was highly associated with the development of depression and bipolar disorder in adulthood. ${ }^{8}$ We now publish data by Roceri et al (pages 609-616) demonstrating that in rats, early maternal deprivation ( $24 \mathrm{~h}$ of maternal separation on day 9) leads to decreased hippocampal levels of BDNF after the animals reach adulthood. The authors also demonstrate that the modulation of BDNF expression in response to an acute stress was altered, indicating a persistent functional impairment in BDNF regulation, possibly compromising the ability to effectively respond to challenging situations. It is interesting that maternal deprivation for just one day, at a critical period in the animal's life, has such persistent long-term effects on BDNF expression and regulation.

Another key finding supporting the role of BDNF in the biology of mood disorders is provided by the work of Sklar et al (pages 579-593), which shows that the BDNF gene is a risk locus for depression. This was a remarkable collaborative effort from some of the world's leading centers in genetics, including Harvard Medical School, Whitehead Institute/MIT Center for Genome Research, Johns Hopkins University, and the Universities of Birmingham and Wales in the UK. Those investigators examined 136 parent-proband trios with bipolar disorder. They studied 76 candidate genes by genotyping 90 SNPs in those genes, and followed those studies with replication in independent populations. This large effort yielded only BDNF as a potential risk gene for bipolar disorder in both genotyping of individual SNPs and haplotype analysis. The authors identified in the region surrounding this gene an undertransmitted haplotype that is associated with bipolar disorder and is marked by a missense mutation in a highly conserved portion of the coding region that may have functional consequences and a mutation in the 3'UTR. It is particularly noteworthy that of 76 candidate genes involving a variety of neurobiological systems, only the BDNF gene emerged a potential risk locus after genotyping and haplotyping studies in the original trios and in a replication sample.

The combination of previously published data and these two new articles strongly support a role for BDNF in the regulation of the stress response and in the biology of mood disorders.

J Licinio and M-L Wong UCLA Laboratory of Pharmacogenomics E-mail: licinio@ucla.edu

\footnotetext{
1 Spina MB et al. J Neurochem 1992; 59: 99-106.

2 Smith MA et al. J Neurosci 1995; 15: 1768-1777.

3 Smith MA et al. Endocrinology 1995; 136: 3743-3750.

4 Shirayama Y et al. J Neurosci 2002; 22: 3251-3261.

5 Frerking $\mathrm{M}$ et al. J Neurophysiol 1998; 80: 3383-3386.

6 Chen B et al. Biol Psychiatry 2001; 50: 260-265.

7 Karege F et al. Psychiatry Res 2002; 109: 143-148.

8 Agid O et al. Mol Psychiatry 1999; 4: 163-172.
} 